Recepción: 23 / 03 / 2018

Aceptación: 17 / 05 / 2018

Publicación: 05 / 06 / 2018
Ciencias Técnicas y Aplicadas

Artículo de Investigación

\title{
Diseño instruccional interactivo Modelo ADDIE durante el proceso de enseñanza - aprendizaje por docentes del Centro Educativo Matriz "Pull Chico"
}

Design interactive instructional model addie during the process of teaching learning by teachers of the school parent "pull guy"

Design instrucional interativo do Modelo ADDIE durante o processo de ensino aprendizagem por professores do Centro de Educação Parental "Pull Chico"

Patricia L. Gallegos-Murillo ${ }^{\mathrm{I}}$ p_gallegos@espoch.edu.ec

Norma V. Cárdenas-Mazón II verónica2012cardenas@hotmail.com

Mónica R. Gallegos-Murillo III monica.gallegos@cordillera.edu.ec

Mayra E. Cáceres-Mena IV macaceresmena@gmail.com

Cecilia T. Limaico-Nieto ${ }^{\mathrm{v}}$ cecilia.limaico@espoch.edu.ec

\section{Correspondencia: p_gallegos@espoch.edu.ec}

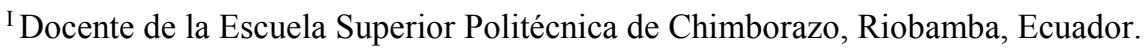

${ }^{\text {II }}$ Docente de la Escuela Superior Politécnica de Chimborazo, Riobamba, Ecuador.

III Instituto Tecnológico Superior Cordillera-Carrera de Optometría, Quito, Ecuador.

IV Docente de la Escuela Superior Politécnica de Chimborazo, Riobamba, Ecuador.

${ }^{\mathrm{V}}$ Docente de la Escuela Superior Politécnica de Chimborazo, Riobamba, Ecuador.
} 


\section{Resumen}

El diseño de un programa de capacitación basado en las competencias TICS para el desarrollo profesional de los docentes, permite mejorar su proceso de enseñanza - aprendizaje a partir de la incorporación de las mismas. A tal efecto, este artículo se propuso como objetivo desarrollar competencias para la gestión de la información, comunicación y estilos de aprendizajes a través de la capacitación del personal del Centro Educativo Matriz "Pull Chico" del cantón Guamote, provincia de Chimborazo, en herramientas Web 2.0, para ello se ejecutó el proceso sistemático interactivo de Diseño instruccional Modelo ADDIE, que consta de cinco etapas: Análisis, Diseño, Desarrollo, Implementación y Evaluación. La metodología fue de tipo cuali cuantitativa, con un enfoque epistémico interpretativo y de tipo descriptivo. La población la constituyeron 17 docentes adscritos al Centro Educativo Matriz "PULL CHICO". El análisis de los datos se realizó cotejeando la información obtenida con lo previsto en las teorías que sustentaron el trabajo. Del análisis e interpretación de los resultados se determinó que el $91 \%$ de los docentes desarrollaron competencias para la gestión de la información, comunicación y estilos de aprendizajes después de participar en la capacitación. Entre sus conclusiones se pudo constatar que el $100 \%$ de los docentes han incorporado con frecuencia las tecnologías de información y comunicación para alcanzar los estándares curriculares confirmando que, el uso de las mismas ha permitido el desarrollo de nuevas estrategias pedagógicas que enriquecen los procesos de enseñanza- aprendizaje.

Palabras clave: herramientas web 2.0; desarrollo de competencias; gestión de la información; estilos de aprendizaje y capacitación en herramientas web 2.0.

\section{Abstract}

The design of a training program based on ICT competences for the professional development of teachers, allows improving their teaching - learning process from the incorporation of them. To this end, this article aimed to develop competencies for the management of information, communication and learning styles through the training of the staff of the Educational Center "Pull Chico" of the Guamote canton, Chimborazo province, in Web tools 2.0, for this, the interactive systematic process of Instructional Design Model ADDIE was executed, which consists of five stages: Analysis, Design, Development, Implementation and Evaluation. The 
methodology was qualitative - quantitative, with an interpretive and descriptive epistemic approach. The population was made up of 17 teachers assigned to the Parent Education Center "PULL CHICO". The analysis of the data was carried out by comparing the information obtained with the foreseen in the theories that supported the work. From the analysis and interpretation of the results, it was determined that $91 \%$ of the teachers developed competencies for the management of information, communication and learning styles after participating in the training. Among its conclusions, it was found that $100 \%$ of teachers have frequently incorporated information and communication technologies to reach the curricular standards confirming that their use has allowed the development of new pedagogical strategies that enrich teaching processes - learning.

Keywords: web 2.0 tools, skills development, information management, learning styles and training on web 2.0 tools.

\section{Resumo}

O desenho de um programa de treinamento baseado em competências em TIC para o desenvolvimento profissional de professores, permite melhorar seu processo de ensino aprendizagem a partir da incorporação dos mesmos. Para o efeito, este artigo foi proposta visa o desenvolvimento de habilidades para a gestão de informação, comunicação e estilos de aprendizagem através da formação de pessoal Centro de Educação Matrix "Pull Chico" Canton Guamote, província de Chimborazo, ferramentas Web 2.0, para isso, foi executado o processo sistemático interativo do Modelo de Design Instrucional ADDIE, que consiste em cinco etapas: Análise, Design, Desenvolvimento, Implementação e Avaliação. A metodologia foi qualitativa quantitativa, com abordagem epistêmica interpretativa e descritiva. A população foi composta por 17 professores designados para o Centro de Educação dos Pais "PULL CHICO". A análise dos dados foi realizada por meio da comparação das informações obtidas com o previsto nas teorias que embasaram o trabalho. A partir da análise e interpretação dos resultados, determinou-se que 91\% dos professores desenvolveram competências para o gerenciamento dos estilos de informação, comunicação e aprendizagem após a participação no treinamento. Entre as suas conclusões, verificou-se que $100 \%$ dos professores têm incorporado frequentemente tecnologias de informação e comunicação para atender aos padrões curriculares, confirmando que a sua 
utilização tem permitido o desenvolvimento de novas estratégias de ensino que melhoram o ensino - aprendendo

Palavras chave: ferramentas da web 2.0; desenvolvimento de competências; gerenciamento de informações; estilos de aprendizagem e treinamento em ferramentas da web 2.0.

\section{Introducción}

Para Actualmente se vive la década de la comunicación y el conocimiento, gracias a la tecnología, las redes han incidido de manera firme y creciente en todos los campos, la educación no es la excepción y ha venido experimentando cambios desde algunos años atrás. Las herramientas de la Tecnología Información Comunicación (TICS) han favorecido en la manera de relacionarnos entre los actores educativos en el proceso de enseñanza aprendizaje, generando la necesidad de reestructurar las políticas educativas nacionales direccionadas a desarrollar las capacidades y competencias indispensables para la vida.

El Centro Educativo Matriz Pull Chico, cuenta con el equipamiento tecnológico, el mismo que no se puede emplear para mediar el proceso de enseñanza aprendizaje, por falta de habilidades en el uso pedagógico de estas herramientas en la gestión educativa, conscientes del reto educativo se pretende orientar a los docentes a través de una capacitación en el desarrollo de competencias digitales mismo que genere la adopción de nuevos estilos de aprendizajes basados en técnicas y metodologías tecnológicas, y sean protagonistas en el uso responsable y eficiente de las TICS aportando y favoreciendo en la calidad educativa dentro del marco de la década de la red. Considerando necesaria la capacitación del 100\% del personal docente en informática básica y herramientas de la Web 2.0.

En este estudio, se consideraron los Estándares de la Unesco sobre competencia en tics para docentes, entendiendo que esta propuesta ofrece orientaciones y directrices para planear programas de capacitación con miras a mejorar la práctica de los docentes en todas las áreas de su desempeño profesional, combinando las competencias en TICS con innovaciones pedagógicas, el plan de estudios (currículo) y la organización escolar. Este modelo propone tres enfoques complementarios estructurados en módulos de competencias. Sin embargo, cada enfoque tiene otros cinco componentes que son; la pedagogía, la práctica y formación profesional de docentes, el plan de estudios (currículo) y evaluación, la organización y administración de la institución 
educativa y la utilización de las TIC, dichos enfoques se pueden aplicar tomando en cuenta las necesidades, visiones y políticas educativas del Sistema Educativo. Todo lo anterior sirvió para diseñar el programa de capacitación basado en las competencias TICS para el desarrollo profesional enfocadas a orientar a los docentes, en el proceso de integración de las mismas en el quehacer educativo, el método y selección de herramientas se fundamentó en los Estándares de Competencias en TICS para docentes de la UNESCO, en la que encontramos directrices para la formación profesional docente con miras a insertar estas tecnologías en la práctica docente

\section{Metodología}

La Metodología fue de tipo cuali -cuantitativa, con un enfoque epistémico interpretativo y de tipo descriptivo, tal como lo expresa Hernández y Fernández (2010), son estudios que describen el fenómeno tal cual como se presenta sin modificar sus cualidades y condiciones. El instrumento que permitió generar información acerca del perfil de los docentes, y conocer sus datos socio académicos fue una encuestas online denominada Chaea y como método sistémico el Diseño instruccional interactivo del Modelo ADDIE, que presenta Sharif y Cho (2015), que comienza por identificar las metas instruccionales y termina con una evaluación, el cual consta de cinco etapas durante su proceso: Análisis, Diseño, Desarrollo, Implementación y Evaluación, esta última se realizó en cada una de sus etapas de manera permanente, lo que facilito depurar errores en el desarrollo de la investigación y regresar a la etapa previa en caso de ser necesario. La población la constituyeron 17 docentes adscritos al Centro Educativo Matriz "PULL CHICO”. El análisis de los datos se realizó cotejeando la información obtenida con lo previsto en las teorías que sustentaron el trabajo.

\section{Desarrollo}

\section{Tipos de aprendizajes}

Al respecto, Kuklinski, (2007), sostiene que el conocimiento se genera bajo una continua negociación y no será producido hasta que los intereses de varios actores estén incluidos. El mismo autor enuncia cuatro tipos de aprendizaje: 
Aprender haciendo. Se basa en las herramientas que incentivan la lectura y la escritura en la Web, se trabajaría entonces bajo el principio de ensayo-error. Por ejemplo: para aprender sobre un determinado tema, generando presentaciones en línea, luego el profesor lo revisa y corrige, este proceso ayudaría a un aprendizaje individual y colectivo a la vez con una tendencia constructivista. Algunas herramientas Web 2.0 que aportan a este tipo de aprendizaje son: Ofimática sobre Web, googledocs, slideshare.

Aprender interactuando. Consiste en la facilidad de intercambio de información de gestión de contenidos, de tal forma que la tarea de intercambio de ideas sea simple. Por ejemplo: el dar un comentario a un artículo determinado de un blog, o de un periódico en línea, una comunicación por voz, etc. Las herramientas que propician este tipo de aprendizaje según Alvear. y Mora (2013) son:

- Herramientas de comunicación tales como: Messenger, yahoo, whatsApp, Facebook, twitter, blog, wiki.

- $\quad$ Herramientas para audio sobre IP como: Skype, Voip, etc;

- Herramientas que permiten dejar comentarios sobre su contenido: video: youtube, de imagen: flickr, picasa, riya, Pireo.; Blogs, Wikis.

- Herramientas de ofimática en línea como: google docs, Ajaxwrite, Writeboard.

Aprender buscando. Constituye una acción previa a la escritura de un documento, por lo que es esencial el poder discriminar la información disponible en el Web, de ahí que resulta fundamental el aprender cómo y dónde buscar contenidos fiables. Siguiendo la línea del mismo autor para hacer referencia a un cuarto aprendizaje incentivado por las herramientas Web 2.0

Aprender compartiendo. El proceso de intercambio de conocimientos y experiencias permite a los educadores participar activamente de un aprendizaje colaborativo. En este sentido, el tener acceso a la información no significa aprender, ya que la abundancia de contenidos existente en la Web no garantiza el aprendizaje. El aprendizaje es concebido a través del intercambio de sus actores, quienes generan, comparten y discuten sus ideas. Para ello la Web 2.0 se ha encargado de abrir los espacios para la discusión e intercambio de ideas, es así que se han creado grandes redes de conocimientos en las que los usuarios, participan activamente. Las herramientas para 
generación de contenido colaborativo, que propician este tipo de aprendizajes, son las wikis, procesadores de texto en línea, podcast, video cast.

Web 2.0 en el proceso de enseñanza - aprendizaje

La Web 2.0 se lleva implantando desde hace varios años en el proceso educativo, más concretamente, a través de la Escuela 2.0, el cual no está siendo aplicado adecuadamente en la mayoría de los casos, desde los distintos centros educativos y profesionales que trabajan en ello. No se debe entender la introducción de la Web 2.0 como una serie de recursos hardware y software que se encaja en la escuela y se presenta a los estudiantes; su inserción debe ir un paso más adelante, estableciendo una serie de aspectos que van desde la actitud positiva hacia la tecnología. Para implantar la Web 2.0 dentro del proceso de enseñanza - aprendizaje y se sugiere los siguientes aspectos:

Cambio de rol del educador. El docente debe pasar de orador de conocimientos a diseñador y orientador en entornos donde se integre las TICS en el aula. (Llorente Cejudo, 2010).

De la enseñanza tradicional al creador de conocimientos e investigador. Se recomienda dejar de lado el momento en el que el profesorado emite sus conocimientos a los estudiantes que escuchaban atentos como simples receptores, a motivar y organizar los conocimientos, fomentando el constructivismo y la investigación por parte de los discentes.

Cambio de metodología y estilos de aprendizaje. Es fundamental a la hora de implantar la Web 2.0 en el proceso de enseñanza-aprendizaje que cambiemos las metodologías y generemos nuevos estilos de aprendizaje. De nada sirve tener muchos recursos tecnológicos si el profesorado sigue utilizando las técnicas tradicionales de aprendizaje. Las políticas educativas, plantea Navarro Jiménez (2008), del Sistema de Educación deben articular concebidos en acciones mediados con la tecnología, el mismo que en nuestros países está en la etapa de implementación. Escenario indispensable en cual debe existir una actitud positiva hacia la tecnología ya que esto motiva al aprendizaje y facilita el desarrollo de habilidades y competencias. Otra política para inducir al docente ecuatoriano en la incorporación de las TICS en la práctica docente son los cursos de formación continua online gratuitos, por tanto, es importante acoger y orientar en la adopción de metodologías para ser parte de este proceso de innovación. 
Desarrollo de nuevas competencias. En la sociedad de la información es preciso fomentar y ser ente activo en el desarrollo de nuevas competencias y destrezas para buscar, recopilar y procesar la información y convertirla en conocimiento significativo.

\section{Estilos de aprendizajes}

Para la comprensión semántica se utilizó la propuesta por Keefe (1982) "llamamos Estilo de Aprendizaje al conjunto de rasgos cognitivos y fisiológicos que sirven como indicadores relativamente estables de cómo perciben los dicentes, interaccionan y responden a su ambiente de aprendizaje" (Ongallo Chanclon, 2005, p.44) se demuestra que las personas piensan, reciben la información, procesan y recuperan de forma diferente.

El entendimiento que tengamos de los estilos de aprendizaje nos permite identificar las diferencias intelectuales de cada estudiante y definir el modo en que aprende. Por tanto, los estilos de aprendizaje representan un elemento valioso que integra el binomio aprendizaje y enseñanza; los cuales facilitan viabilizar al docente en la realización de diagnósticos, estrategias factibles que ratifican tanto el aprendizaje como el desarrollo de habilidades de pensamiento, una de las ventajas sobresalientes de conocer el propio estilo de aprendizaje de los dicentes es que aprenden con más efectividad.

Una vez analizado los distintos estilos de aprendizaje propuesto por varios autores, se ha llegado a la conclusión de que si un estudiante posee más actitudes en uno de estos estilos de aprendizaje tendera a favorecer una manera de aprendizaje propia. Siendo la siguiente propuesta por Rosero (2008), en la que destaca los siguientes estilos de aprendizajes:

Estilo Activo. Son de mente abierta, emprenden nuevas tareas con entusiasmo. Son improvisadores asiduos, son abiertos a las nuevas tecnologías. Les cuesta trabajo aprender cuando tienen que asimilar, analizar, e interpretar los datos.

Estilo Reflexivo. Indaga con exhaustividad el porqué de las situaciones. Antepone la reflexión a la acción, consideran y observan las experiencias desde diferentes perspectivas. Recogen datos, analizándolos con detenimiento antes de llegar a alguna conclusión. Son personas que gustan considerar todas las alternativas posibles antes de realizar una acción. 
Estilo teórico. Cuestionan las nuevas versiones que aparecen, antes de abandonar la teoría conocida, buscando la lógica en los problemas para establecer principios. Adaptan e integran las observaciones dentro de teorías lógicas y complejas. Tienden a ser perfeccionistas. Integran los hechos en teorías coherentes. Les gusta analizar y sintetizar. Son profundos en su sistema de pensamiento, a la hora de establecer principios, teorías y modelos.

Estilo pragmático. Tiene gusto de las nuevas ideas, por la experimentación y aplicación. Actúan rápidamente $\mathrm{y}$ con seguridad con aquellas ideas y proyectos que les atraen, tienen a ser impacientes cuando las persona teorizan.

La comprensión de las características individuales de aprendizaje, expresa Christian y Lino (2016), permiten definir las diferencias cognitivas de cada estudiante, de modo que se pueda identificar la forma en que el discente pueda construir el conocimiento, de ahí nace la importancia de seleccionar los recursos tecnológicos en función de los procesos de aprendizaje a utilizarse, los mismos que permitan maximizar el aprovechamiento de los recursos tecnológicos. Son variados los recursos Web 2.0, explica Larequi, (2015), que se puede encontrar para fines pedagógicos, para que los docentes y estudiantes pueden crear y compartir sus propios contenidos con calidad, versatilidad y amplitud. Los mismos que motivan la inserción de nuevas metodologías y de planteamientos didácticos innovadores, permitiendo la colaboración entre iguales, el altruismo, la inteligencia colectiva, la creación y difusión de conocimientos compartidos, la responsabilidad individual y la participación social e institucional.

\section{Metodología}

La metodología fue de tipo cuali -cuantitativa, con un enfoque epistémico interpretativo y de tipo descriptivo, tal como lo expresa Hernández y Fernández (2010), son estudios que describen el fenómeno tal cual como se presenta sin modificar sus cualidades y condiciones. El instrumento que permitió generar información acerca del perfil de los docentes, y conocer sus datos socio académicos fue una encuestas online denominada Chaea y como método sistémico el Diseño instruccional interactivo del Modelo ADDIE, que presenta Sharif y Cho (2015), que comienza por identificar las metas instruccionales y termina con una evaluación, el cual consta de cinco etapas durante su proceso: Análisis, Diseño, Desarrollo, Implementación y Evaluación, esta última se realizó en cada una de sus etapas de manera permanente, lo que facilito depurar errores 
en el desarrollo de la investigación y regresar a la etapa previa en caso de ser necesario. La población la constituyeron 17 docentes adscritos al Centro Educativo Matriz "PULL CHICO”. El análisis de los datos se realizó cotejeando la información obtenida con lo previsto en las teorías que sustentaron el trabajo.

\section{Resultados}

Se conocieron las preferencias de aprendizajes por parte de los docentes, posterior a la aplicación de la encuesta Chaea, en la que determinó el perfil de los docentes, y conocieron los datos socio académicos de la población en estudio.

Fue posible insertar de manera progresiva las herramientas tecnológicas en la gestión de la información, comunicación, renovación de estilos de aprendizajes apoyados en competencias digitales en el proceso de enseñanza aprendizaje, durante la definición del programa de capacitación tomando como guía las directrices de los Estándares de Competencias en TICS para docentes dadas por la Unesco del Enfoque Nociones Básicas en TICS.

En la fase desarrollo del programa se elaboraron los contenidos, recursos y materiales a ser utilizados en la capacitación para el total de la población. En la implementación se puso en práctica la acción formativa del plan de capacitación basada en metodologías activas y herramientas Web 2.0, en la cual la evaluación estuvo presente durante todo el proceso facilitando la corrección de errores desde cualquier fase permitiendo alcanzar el objetivo propuesto con menos errores.

Los docentes manifestaron que los estudiantes han adoptado un nuevo perfil basando su aprendizaje en la Web 2.0, que los vincula de forma casi obligada a adoptar nuevas maneras de aprender y de relacionarse con el conocimiento y con la información, ayudándoles a entender y a dialogar didácticamente mejor con los estudiantes en búsqueda de un verdadero Entorno Social de Aprendizaje.

Fue posible constatar que la cultura digital e interactiva está creciendo de manera global, por tanto, los docentes han adoptado un nuevo perfil que los vincula casi obligadamente adoptar nuevas maneras de aprender y de relacionarse con el conocimiento y con la información ya que éstos les ayudan a entender y a dialogar didácticamente mejor con sus estudiantes. 


\section{Conclusiones}

Es viable en nuestro medio el uso de los Estándares de la Unesco en materia de competencias en TICS para docentes, ya que contiene directrices que los orientan a integrar las TIC en sus actividades de enseñanza y aprendizaje, y cuyo punto de partida es la correlación de lo que sabe y aplica el docente.

El total de los docentes capacitados han incorporado con frecuencia las tecnologías de información y comunicación para alcanzar los estándares curriculares confirmando que, el uso de las mismas ha permitido el desarrollo de nuevas estrategias pedagógicas que han enriquecido los procesos de aprendizaje.

El 59\% de los docentes utilizan las herramientas Web 2.0 para apoyar su labor educativa y el resto representado por el $41 \%$ lo utiliza a veces ya que depende del grupo de estudiantes y el nivel con los que diariamente ejecuta las actividades académicas.

Las herramientas Web 2.0 de mayor preferencia de uso entre las herramientas de comunicación son el blog, wikis, google docs, YouTube. De los cuales se determinó que YouTube es utilizado en el $76 \%$ por los estudiantes de Educación General Superior y Bachillerato para reforzar y ampliar los conocimientos, por la utilidad en la generación y ampliación de contenidos; mientras que Google Docs en el 59\% en empleado para realizar actividades colaborativas con sus pares ayudándoles a estimular habilidades personales, disminuir los sentimientos de aislamiento y propiciar resultados de grupo a partir de la participación individual.

En la asignatura que los docentes han empleado con más frecuencia las herramientas Web 2.0 es Ciencias Naturales en un $41 \%$, para comprender los conceptos fundamentales de esta ciencia, su uso ha propiciado que se generen nuevas estrategias pedagógicas facilitando a los estudiantes interactuar en contextos virtuales, recursos multimedia, simulaciones, enciclopedias virtuales, etc. Recalcando que éstas herramientas facilitan experiencias que cautivan el interés en todas las asignaturas y para todos los niveles educativos.

Se observa que los docentes se sienten confiados para llevar el control y mantener los de registros de asistencia y calificaciones de sus estudiantes en línea a través de la plataforma virtual educa Ecuador, integrándose de esta manera a un espacio virtual. 
En cuanto a los estudiantes, se observó que presentaron interés y motivación por realizar las actividades haciendo uso las herramientas Web 2.0, así como también muestran mayor sentido de solidaridad y colaboración con sus compañeros para realizar las actividades propuestas.

\section{Referencias Bibliográficas}

Alvear, A. y Mora, P. (2013). Herramientas Web2.0 y Estilos de aprendizaje.

Ciencia, tecnología y sociedad, 82.

Christian, C., y Lino, E (2016). Aplicaciones de los entornos virtuales en las aulas universitarias.

Hernández, R; Fernández, C y Baptista, P. (2010). Metodología de la investigación. Quinta Edición. Editorial: McGraw-Hill. México.

Kuklinski, C. (2007). Planeta WEB 2.0 Inteligencia Colectiva o Medios Fast Food. México: Recerca d"interccion Digital.

Larequi, E. (2015). La Web 2.0 en el ámbito educativo. Propuestas TIC para el área de Lengua Materiales TIC para el Máster en Formación del Profesorado, 1.

llorente Cejudo, M. d. (2010). Nuevos escenarios desde la perspectiva de la Web 2.0. Gabinete de Comunicación y Educación, 6-8.

Navarro Jimenez, M. (2008). Como diagnosticar y mejorar los estilos de aprendizaje. Almeria: Asociacion Procompal.

Ongallo Chanclon, C. (2005). Aplicaciones Educativas de las tecnologías de la información y la comunicación. Santader: Egraf s.a.

Sharif, A., y Cho, S. (2015). Modelos de diseño instruccional. Diseñadores instruccionales del siglo XXI: cruzando las brechas perceptuales entre la identidad, práctica, impacto y desarrollo profesional, 74-75. 